\title{
A study of the s-process in the carbon-rich post-AGB stars IRAS 06530-0213 and IRAS 08143-4406 on the basis of VLT-UVES spectra ${ }^{\star, \star \star}$
}

\author{
M. Reyniers ${ }^{1}$, H. Van Winckel ${ }^{1, \star \star \star}$, R. Gallino ${ }^{2,3}$, and O. Straniero ${ }^{4}$ \\ ${ }^{1}$ Instituut voor Sterrenkunde, Departement Natuurkunde en Sterrenkunde, K.U. Leuven, Celestijnenlaan 200B, 3001 Leuven, \\ Belgium \\ 2 Dipartimento di Fisica Generale, Universitá di Torino, via Pietro Giuria 1, 10125 Torino, Italy \\ 3 Centre for Stellar and Planetary Astrophysics, School of Mathematical Sciences, Monash University, 3800 Australia \\ ${ }^{4}$ Osservatorio Astronomico di Collurania, 64100 Teramo, Italy
}

Received 25 September 2003 / Accepted 18 December 2003

\begin{abstract}
In an effort to extend the still limited sample of s-process enriched post-AGB stars, high-resolution, high signalto-noise VLT+UVES spectra of the optical counterparts of the infrared sources IRAS 06530-0213 and IRAS 08143-4406 were analysed. The objects are moderately metal deficient by $[\mathrm{Fe} / \mathrm{H}]=-0.5$ and -0.4 respectively, carbon-rich and, above all, heavily s-process enhanced with a [1s/Fe] of 1.8 and 1.5 respectively. Especially the spectrum of IRAS 06530-0213 is dominated by transitions of s-process species, and therefore resembling the spectrum of IRAS $05341+0852$, the most s-process enriched object known so far. The two objects are chemically very similar to the $21 \mu \mathrm{m}$ objects discussed in Van Winckel \& Reyniers (2000). A homogeneous comparison with the results of these objects reveals that the relation between the third dredgeup efficiency and the neutron nucleosynthesis efficiency found for the $21 \mu \mathrm{m}$ objects, is further strengthened. On the other hand, a detailed comparison with the predictions of the latest AGB models indicates that the observed spread in nucleosynthesis efficiency is certainly intrinsic, and proves that different ${ }^{13} \mathrm{C}$ pockets are needed for stars with comparable mass and metallicity to explain their abundances.
\end{abstract}

Key words. stars: AGB and post-AGB - stars: abundances - stars: carbon - stars: individual: IRAS 06530-0213 stars: individual: IRAS 08143-4406 - stars: chemically peculiar - stars: evolution - line: identification

\section{Introduction}

In the last two decennia, the progress in the theoretical modelling of AGB stars, as well as the qualitative and quantitative improvement of the observational data of (post-)AGB stars, are impressive. Whereas, before the launch of the IRAS satellite, only a few post-AGB candidates were known, the present sample consists of about 220 objects (Szczerba et al. 2001). The main keyword, however, that can be applied to the postAGB sample is diversity. In practically all their aspects, post-AGB stars are much more diverse than theoretically anticipated.

Send offprint requests to: $\mathrm{M}$. Reyniers,

e-mail: Maarten. Reyniers@ster.kuleuven.ac.be

* Based on observations collected at the European Southern Observatory, Paranal, Chile (ESO Programme 66.D-0171).

$\star \star$ Table 4 is only available in electronic form at the CDS via anonymous ftp to cdsarc.u-strasbg.fr $(130.79 .128 .5)$ or via http://cdsweb.u-strasbg.fr/cgi-bin/qcat?J/A+A/417/269 $\star \star \star$ Postdoctoral fellow of the Fund for Scientific Research, Flanders.
In the case of the morphology, the resolved post-AGB stars display a surprisingly wide variety of shapes and structures. There are only few sources displaying a spherically symmetric morphology, most sources possessing a clear bipolar structure (see e.g. Sahai 2001). The aspherical structure was already demonstrated earlier by ground based observations both in optical images of the scattered visible light (e.g. Hrivnak et al. $1999, R \sim 0$ '.75), and in mid-IR images of the thermal emission of dust (e.g. Meixner et al. 1999, $R \sim 1^{\prime \prime}$ ). Many more structural details were, however, resolved on HST images (e.g. Ueta et al. 2000; Hrivnak et al. 2001). For a recent review, we refer to Balick \& Frank (2002).

Concerning the circumstellar chemistry, the high resolution spectroscopy of the infrared excess also reveals an interesting chemical evolution. The observations made by the Infrared Space Observatory (ISO) satellite brought important progress in this domain. In an O-rich chemistry, the dust is mainly composed of amorphous silicates, but also bands of crystalline silicates are found. In the carbon dominated chemistry, the dust consists mainly of amorphous carbon, $\mathrm{SiC}$, and probably $\mathrm{MgS}$ (the carrier of the broad $30 \mu \mathrm{m}$ band, Hony et al. 2002). Also the "Unidentified InfraRed" (UIR) bands are 


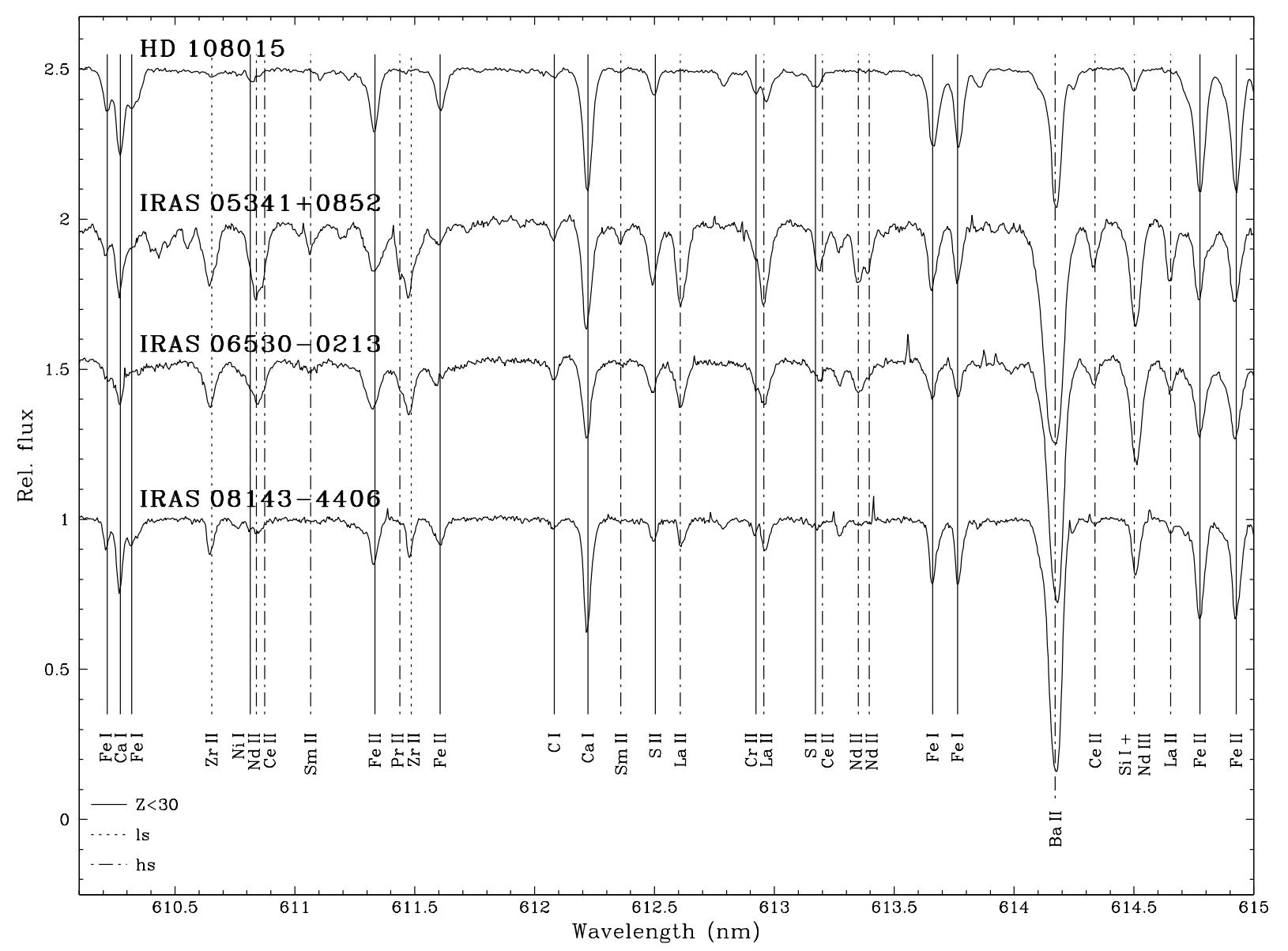

Fig. 1. The spectra of IRAS 06530-0213 and IRAS 08143-4406 compared with the heavily s-enriched post-AGB star IRAS $05341+0852$ and the non s-enriched post-AGB star HD 108015. IRAS 05341+0852 is discussed in Paper I, and it is the most heavily s-process enriched star known so far. HD 108015 has been analysed by Van Winckel (1997) and has atmospheric parameters comparable with the other three ( $T_{\text {eff }}$, $\left.\log g, \xi_{\mathrm{t}}\right)=\left(7000 \mathrm{~K}, 1.5,4.0 \mathrm{~km} \mathrm{~s}^{-1}\right)$ but is slightly less metal deficient $([\mathrm{Fe} / \mathrm{H}]=-0.1)$. A complete line identification of this spectral interval has been pursued by the use of the VALD database (Kupka et al. 1999). Lines of light s-process elements (Sr peak) are identified by a dotted line; lines of heavy s-process elements (Ba peak) by a dash-dotted line; lines of other elements (which are mainly $\alpha$ and iron peak elements) by a full line. The s-process enhancement of the three IRAS stars is clear. IRAS $05341+0852$ is the most s-enriched one, but the stronger lines are also due to a slightly lower temperature of this object. All spectra are VLT+UVES spectra, except the spectrum of HD 108015, which is a ESO1.5+FEROS spectrum taken at March 22, 2000.

seen in these IR spectra, commonly attributed to Polycyclic Aromatic Hydrocarbons (PAHs). A sub-class of C-rich postAGB stars shows a feature at $21 \mu \mathrm{m}$, discovered by Kwok et al. (1989), possibly attributed to TiC nanocrystals (von Helden et al. 2000).

Finally, there is an intriguing diversity in the observed photospheric chemical patterns of the post-AGB stellar sample. The third dredge-up is expected to bring carbon and freshly synthesized s-process elements to the photosphere of an AGB star. The spectral lines of these elements are thus expected to be strong in the spectra of post-AGB stars. This is indeed what we observe, but remarkably only in a certain subclass of post-AGB objects. There are very similar objects (with a comparable mass and metallicity) that do not show these enhancements. This chemical dichotomy is surprisingly strict in the sense that an object is either severly enriched, or it is not enriched at all (even s-process deficiencies are observed). This is illustrated in Fig. 3 of Van Winckel (2003) on which the overabundance of the s-process element zirconium (atomic number
$Z=40$ ) is plotted against metallicity for a sample of stars that all show similar characteristics for a genuine post-AGB classification, like a double peaked SED, low metallicity and kinematics pointing to membership of an old population. The chemical difference is directly clear from the spectrum itself and we refer to Fig. 1 for an illustration. Moreover, all s-enriched objects studied till now are suspected or confirmed $21 \mu \mathrm{m}$ sources. Six of these sources were recently analysed in a homogeneous abundance study by Van Winckel \& Reyniers (2000), hereafter Paper I. In the non s-enriched objects (see e.g. Luck et al. 1990; Van Winckel 1997, for abundance analyses of these objects), no enrichments were found larger than the $[\mathrm{Zr} / \mathrm{Fe}] \sim 0.2$ error level.

In the present study, we present the analysis of two newly discovered s-process enriched post-AGB stars, IRAS 06530-0213 and IRAS 08143-4406 (Table 1). Despite the fact that these IRAS sources have been both candidate post-AGB stars for more than a decade (as derived from their position in the IRAS color-color diagram, e.g. 
Table 1. Basic parameters of the two objects discussed in this study.

\begin{tabular}{|c|c|c|c|c|c|c|c|c|c|c|c|}
\hline \multirow[t]{3}{*}{ IRAS } & \multirow{2}{*}{\multicolumn{2}{|c|}{$\begin{array}{c}\text { Equatorial } \\
\text { coordinates }\end{array}$}} & \multirow{2}{*}{\multicolumn{2}{|c|}{$\begin{array}{c}\text { Galactic } \\
\text { coordinates }\end{array}$}} & \multirow{2}{*}{\multicolumn{2}{|c|}{$\begin{array}{c}\text { Visual } \\
\text { magnitude }\end{array}$}} & \multirow{3}{*}{$\begin{array}{l}\text { Spectral } \\
\text { Type }\end{array}$} & \multicolumn{4}{|c|}{ IRAS Fluxes (Jy) } \\
\hline & & & & & & & & \multirow[t]{2}{*}{$f_{12}$} & \multirow[t]{2}{*}{$f_{25}$} & \multirow[t]{2}{*}{$f_{60}$} & \multirow[t]{2}{*}{$f_{100}$} \\
\hline & $\alpha_{2000}$ & $\delta_{2000}$ & 1 & $\mathrm{~b}$ & $m(\mathrm{~b})$ & $m(\mathrm{v})$ & & & & & \\
\hline \multirow[t]{2}{*}{$06530-0213$} & 065532.1 & -021730 & 215.44 & -0.13 & $16.4^{a}$ & $14.0^{a}$ & $\mathrm{~F} \mathrm{I}^{a}$ & 6.11 & 27.41 & 15.05 & 4.10 \\
\hline & & & & & $16.3^{b}$ & $14.1^{b}$ & F5 I ${ }^{c}$ & & & & \\
\hline $08143-4406$ & 081602.9 & -441601 & 260.83 & -5.07 & $14.1^{b}$ & $12.4^{b}$ & $\mathrm{~F} 8 \mathrm{I}^{b}$ & 0.60 & 9.26 & 6.06 & $<3.73$ \\
\hline
\end{tabular}

Source SIMBAD, except: ${ }^{a}$ Slijkhuis (1992), ${ }^{b}$ Reddy \& Parthasarathy (1996), ${ }^{c}$ Hrivnak \& Reddy (2003)

Preite-Martinez 1988), they are both still poorly studied. For IRAS 06530-0213 this can partly be attributed to the optical weakness of this source (Table 1), while for IRAS 081434406 it is less clear why this source has been neglected for such a long time. Based on a low resolution spectrum $\left(10.7 \AA\right.$ pixel $\left.^{-1}\right)$ taken with the $2.5 \mathrm{~m}$ INT on La Palma, Slijkhuis (1992) attributed a spectral type F0I to the optical counterpart of IRAS 06530-0213. In a very recent study, Hrivnak \& Reddy (2003) found that this is a too early spectral type and classified IRAS 06530-0213 as a F5 supergiant. The spectral type of IRAS 08143-4406 (F8I) was taken from Reddy \& Parthasarathy (1996) and is based on a low resolution (5.7 A pixel ${ }^{-1}$ ) spectrum taken with a $1 \mathrm{~m}$ telescope.

The paper is organised as follows: in Sect. 2 we describe the observations of the two programme stars, together with the data reduction. In Sect. 3 the radial velocities of the two programme stars are compared with literature values, with the aim of testing their possible binary nature. Section 4 focusses on the technical aspects of the analysis, including atmospheric parameter determination, line selection and spectrum synthesis. Also our abundance results are presented in this section. In the next section, we compare the two program stars with the $21 \mu \mathrm{m}$ stars of Paper I. It turns out that the two stars share the same chemical properties with the $21 \mu \mathrm{m}$ stars. Section 6 is devoted to a detailed comparison with theoretical chemical AGB model predictions. In this section, also the $21 \mu \mathrm{m}$ stars are compared with these chemical models. Finally, in Sect. 7 we summarize our most important findings.

\section{Observations}

High resolution, high signal-to-noise VLT+UVES spectra of the two programme stars are taken in the framework of our ongoing program to study the photospheric chemical composition of stars in their last stages of evolution (e.g. Paper I; Reyniers \& Van Winckel 2001, 2003). The two targets are part of a larger sample of eleven post-AGB objects that were observed in service mode during two periods (04-07/2000 and 01-02/2001, see Reyniers 2002). The resolving power of these spectra varies between $\sim 55000$ and $\sim 60000$. Some details about the observations are given in Table 2.

The standard reduction was performed in the dedicated "UVES context" of the MIDAS environment and included bias correction, cosmic hit correction, flat-fielding, background correction and sky correction. We used optimal extraction to convert frames from pixel-pixel to pixel-order space. The spectra were normalised by dividing the individual orders
Table 2. Observational log. Spectral gaps occur between $577 \mathrm{~nm}$ and $583 \mathrm{~nm}$ and between $854.4 \mathrm{~nm}$ and $864.5 \mathrm{~nm}$ due to the spatial gap between the two UVES CCDs.

\begin{tabular}{ccccc}
\hline \hline date & $\begin{array}{c}\text { UT } \\
\text { start }\end{array}$ & $\begin{array}{c}\text { Exp. time } \\
\text { (s) }\end{array}$ & $\begin{array}{c}\text { Wavelength } \\
\text { interval }(\mathrm{nm})\end{array}$ & $S / N$ \\
\hline \multicolumn{5}{c}{ IRAS 06530-0213 } \\
\hline IRAS 08143-4406 \\
\hline $2001-01-12$ & $03: 36$ & $3 \times 1800$ & $477.5-681$ & 100 \\
\hline $2001-01-16$ & $06: 41$ & $2 \times 1800$ & $374.5-498$ & 130 \\
$2001-01-16$ & $06: 41$ & $1 \times 1800$ & $670.5-1055$ & sat. \\
$2001-01-16$ & $07: 16$ & $3 \times 500$ & $670.5-1055$ & 200 \\
$2001-02-01$ & $04: 21$ & $1 \times 1800$ & $477.5-681$ & 150 \\
\hline
\end{tabular}

sat. $=$ saturated.

by a smoothed spline function defined through interactively identified continuum points. Orders were merged after this normalisation. For a detailed description of the reduction procedure, we refer to Reyniers (2002). In Table 2, we also list some indicative signal-to-noise values of the final data product. Sample spectra can be found in Figs. 1, 3 and 5.

\section{Radial velocities}

Since binarity can influence the chemical evolution of a postAGB star drastically (see e.g. Van Winckel et al. 1995), it is important to determine and monitor accurate radial velocities for these objects. The large wavelength coverage together with the high resolution of our UVES spectra permit us to determine these velocities using a large number of lines. The obtained radial velocities are gathered in Table 3, together with the few values found in the literature. An additional velocity is obtained with the CORALIE spectrograph mounted on the Swiss telescope Euler in La Silla, Chile. Highly accurate velocities are obtained with this instrument by cross-correlation observed spectra with a spectrum mask of comparable spectral type.

It is obvious that there is still monitoring needed to draw any final conclusion, but till now, there is no evidence for a binary motion for the two programme stars.

\section{Abundance analysis}

\subsection{Atomic data and atmospheric parameters}

A list of lines useful for the chemical analysis of A and F type stars has been collected at the Instituut voor Sterrenkunde during the past few years and is regularly updated. This list is described in detail in Paper I. A recent update of the list after the 
Table 3. Heliocentric radial velocities of the programme stars, both from the literature and from this study. If the velocity is obtained in this study, the number of lines on which it is based is given in the last column; otherwise the reference is given in the same column. The last velocity is obtained with the CORALIE spectrograph. Typical errors on these velocities are between 1.0 and $1.5 \mathrm{~km} \mathrm{~s}^{-1}$.

\begin{tabular}{lccc}
\hline \hline $\begin{array}{c}\text { Date } \\
\text { (yyyy-mm-dd) }\end{array}$ & $\begin{array}{c}v_{r} \\
\left(\mathrm{~km} \mathrm{~s}^{-1}\right)\end{array}$ & Method $^{\dagger}$ & $\begin{array}{c}\text { Ref.” or number } \\
\text { of used lines }\end{array}$ \\
\hline \multicolumn{4}{c}{ IRAS 06530-0213 } \\
\hline $1991-04$ & 50 & $\mathrm{a}$ & ref. 1 \\
$1997-10-17$ & 51.0 & $\mathrm{~b}$ & ref. 2 \\
$2001-01-12$ & 49.8 & $\mathrm{~b}$ & $\mathrm{n}=236$ \\
$2001-12-10$ & 50.4 & $\mathrm{~b}$ & ref. 2 \\
\hline \multicolumn{5}{c}{ IRAS 08143-4406 } \\
\hline $2001-01-16$ & 51.5 & $\mathrm{~b}$ & $\mathrm{n}=134$ \\
$2001-02-01$ & 52.2 & $\mathrm{~b}$ & $\mathrm{n}=434$ \\
$2002-05-22$ & 49.6 & $\mathrm{c}$ & \\
\hline
\end{tabular}

method a: $\mathrm{CO}(J=2-1)$ line; b: mean of individual optical lines, c: cross-correlation with spectrum mask.

ref. 1: Hu et al. (1994); 2: Hrivnak \& Reddy (2003).

Table 4. Line identifications and measured equivalent widths for IRAS 06530-0213 and IRAS 08143-4406. This table is only available in electronic form at the CDS. It contains the following columns: the rest wavelength (in $\AA$ ), the identification, the lower excitation potential (in $\mathrm{eV}$ ), the adopted $\log g f$ value, the measured equivalent widths for IRAS 06530-0213 (in $\mathrm{m} \AA$ ), the measured equivalent widths for the red setting (2001-02-01, see Table 2) of IRAS 08143-4406 and the measured equivalent widths for the dichroic setting (2001-01-16, see Table 2) of IRAS 08143-4406.

publication of Paper I consists of the inclusion of the data published by Lawler et al. (2001a) for La and Lawler et al. (2001b) for Eu. Using this same list, we do not only restrict ourselves to lines with reliable atomic data, but we also ensure that the different analyses are perfectly homogeneous.

Equivalent widths of unblended lines were measured with direct integration; multiple Gaussian fitting was applied for lines with (a) blended wing(s). The entire line list, together with the measured equivalent widths for both stars can be found in Table 4, which is published in electronic form at the CDS. The atmospheric parameters were obtained by the usual spectroscopic method in which the effective temperature is obtained by imposing the iron abundance, derived from the individual Fe I lines, to be independent of lower excitation potential; the gravity by imposing ionization equilibrium; the microturbulent velocity by imposing iron abundance derived from the individual Fe I lines to be independent of (reduced) equivalent width. The model atmospheres of Kurucz (1993) were used, in combination with the latest version (April 2002) of the LTE abundance calculation routine MOOG (Sneden 1973).

The results on other species can be used as a check for the atmospheric parameters, providing that the number of lines for that species is large enough. Elements for which we detected lines of different ions can be used as a first consistency

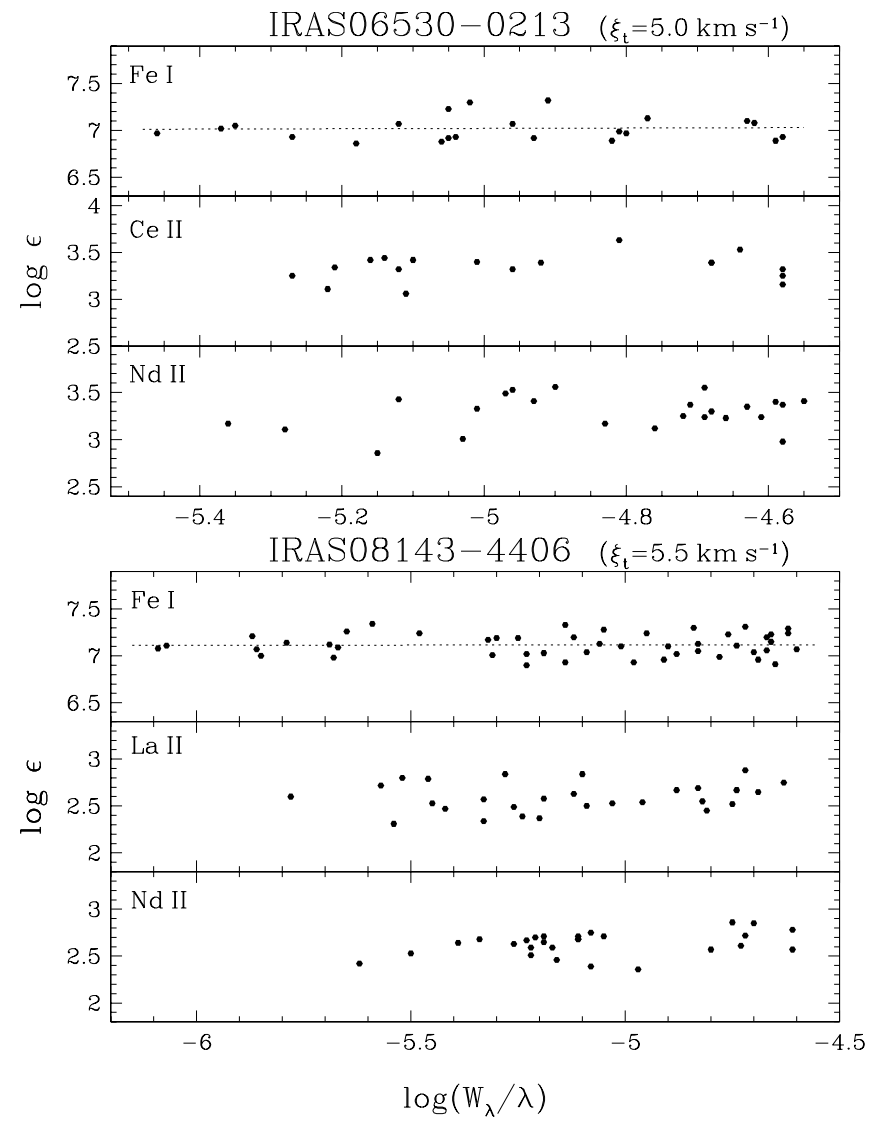

Fig. 2. Reduced equivalent width - abundance diagrams for elements of which the abundance is based on more than fifteen lines. Such diagrams are constructed to derive the microturbulent velocity $\xi_{\mathrm{t}}$ for the model atmosphere; the correct value for $\xi_{\mathrm{t}}$ is found if no trend is seen on the diagram. In this figure, we check if the abundances of some non-iron species are consistent with the value for $\xi_{\mathrm{t}}$ that was derived from a study of the iron lines.

check of the model atmosphere. A temperature check is difficult since for most elements, the range in excitation potential is quite small. Therefore, only the microturbulent velocity parameter $\xi_{\mathrm{t}}$ can be checked in this way. For a given element, the most straightforward method is then to search for that $\xi_{\mathrm{t}}$ for which the slope of the linear fit on a reduced width - abundance diagram equals zero. With this method, however, one loses the particular characteristics of the specific diagram, in the sense that mostly outliers in $\log \left(W_{\lambda} / \lambda\right)$ determine the obtained slope. Therefore, it is often much more instructive to inspect the diagram by eye, than to rely on a least-squares method. This is done in Fig. 2, on which we plotted the Fe, $\mathrm{Ce}$ and $\mathrm{Nd}$ diagrams for IRAS 06530-0213, and the Fe, La and Nd diagrams for IRAS 08143-4406. From this figure it is clear that (a) the spread in reduced width $\log \left(W_{\lambda} / \lambda\right)$ is significantly larger for Fe than for the other elements and that (b) the spread in abundance $\log \epsilon$ is smaller in the case of iron. For the non-iron species in Fig. 2 it is difficult to decide whether a real trend is seen or not. Especially the points in the $\mathrm{Nd}$ diagram display a somewhat confusing pattern. Several authors already stressed the need for new atomic data for Nd II (see e.g. Sneden et al. 2002, for a discussion of the available Nd II data). We can conclude that there 
is no clear indication for a dependence of $\xi_{\mathrm{t}}$ on the species that is used to derive it. Note that an increase of $\sim 3 \mathrm{~km} \mathrm{~s}^{-1}$ does not induce large changes in the derived abundances (a decrease of $\sim 0.1$ dex for the strongest lines in our line list).

A particular problem arose in the determination of the parameters of IRAS 08143-4406. Contrary to IRAS 06530-0213, the star is measured in two different spectrograph settings: on 2001-01-16 the dichroic setting (hereafter DI2) is used with a simultaneous observation in the blue and in the red, while on 2001-02-01 the red setting centered on $580 \mathrm{~nm}$ (hereafter RED580) is observed (see also Table 2). Between these two observations, there is a time gap of 16 days. By inspecting the spectral overlap of the two observations, we concluded that the atmospheric parameters apparently changed during this time gap. The adoption of two different sets of atmospheric parameters for each setting is therefore necessary. Unfortunately, the number of Fe lines suitable for parameter determination is too low for the dichroic setting. An alternative method had to be developed to obtain the parameters for this setting.

We chose to deduce the atmospheric parameters for the 2001-01-16 setting of IRAS 08143-4406 relative to its parameters of the 2001-02-01 setting, by demanding that the lines in the overlap should yield the same abundance. So, after determination of the atmospheric parameters for IRAS 08143-4406 for the 2001-02-01 setting, we searched for the model parameters for the 2001-01-16 setting, by minimizing the abundance difference of the lines in the overlap. This yielded model parameters $\left(T_{\mathrm{eff}}, \log g, \xi_{\mathrm{t}}\right)=\left(7250 \mathrm{~K}, 1.5,5.5 \mathrm{~km} \mathrm{~s}^{-1}\right)$ for the 2001-02-01 setting, and $\left(7050 \mathrm{~K}, 1.2,5.5 \mathrm{~km} \mathrm{~s}^{-1}\right)$ for 2001-01-16. One should note that a difference of $0.3 \mathrm{dex}$ in gravity is rather large: assuming a mass of $1.5 M_{\odot}$, a $\log g$ of 1.5 yields an approximative radius of $R \simeq 36 R_{\odot}$, while a $\log g$ of 1.2 yields $R \simeq 51 R_{\odot}$. This results in a pulsation velocity of $\bar{v} \simeq 7.5 \mathrm{~km} \mathrm{~s}^{-1}$, which is not consistent with the seemingly constant radial velocity of IRAS 08143-4406 (see Table 3). Moreover, such a change in atmospheric parameters would induce a difference of $\sim 0.6$ mag in $\mathrm{V}$ in 16 days. Since the gravity is not well constrained (a typical error in $\log g$ is 0.5 ), we decided to keep the difference in the parameters in order to keep the abundances deduced from the two settings as consistent as possible.

\subsection{Spectrum synthesis of IRAS 06530-0213}

\subsubsection{The macroturbulent broadening $\xi_{\mathrm{m}}$}

When computing a spectrum synthesis, a broadening parameter, additional to the usual atmospheric parameters, is required to match the synthetic line profiles with the observed ones. This broadening is the combined effect of instrumental, macroturbulent and rotational broadening. The latter one is, however, thought to be small in F-G supergiants. Since the instrumental broadening can be deduced from the width of the Th-Ar lines of the calibration spectra, the macroturbulent broadening $\left(\xi_{\mathrm{m}}\right)$ is the only parameter that has to be estimated before comparing synthetic spectra with observed ones. We concentrated on the spectra of IRAS 06530-0213, as this star is the most interesting in the framework of s-process nucleosynthesis.

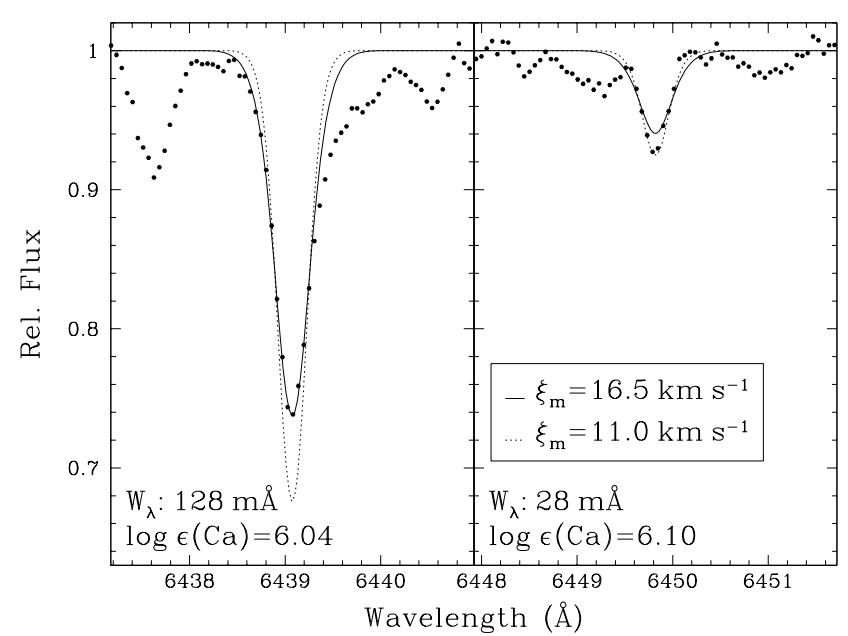

Fig. 3. Synthesis of two lines of neutral calcium. From this figure, it is clear that spectral lines cannot be fitted using one fixed value for the macroturbulent broadening $\xi_{\mathrm{m}}$. The synthesis is made using the abundance as derived from their equivalent width. The two abundances are in good agreement. The best-fit macroturbulent broadening, however, differs by $5.5 \mathrm{~km} \mathrm{~s}^{-1}$.

For the instrumental broadening we took the median of the resolution of the Th-Ar lines used in the calibration, which is $\delta \lambda=\lambda / 59525 \AA$ for the lower (EEV) CCD and $\delta \lambda=\lambda / 56575 \AA$ for the upper (MIT) CCD. The macroturbulent velocity $\xi_{\mathrm{m}}$ was then obtained as follows. We selected 121 unblended lines from the abundance analysis of IRAS 06530-0213 from different species and ionisation (neutral as well as singly ionised). Each of these 121 lines was synthesized with MOOG using the abundance as derived by its equivalent width. The macroturbulent broadening $\xi_{\mathrm{m}}$ is the only free parameter in this synthesis. We fitted the synthetic spectra by varying this parameter in steps of $0.5 \mathrm{~km} \mathrm{~s}^{-1}$. The profile used for the macroturbulent broadening is a radial-tangential macroturbulence profile based on the work of Gray (1992). During this fitting procedure, we soon realized that we could not fit the lines with one fixed value for $\xi_{\mathrm{m}}$, but that each line required its own value. This is illustrated in Fig. 3, on which we synthesized two calcium lines. These lines are lying in the same spectral region and the abundances derived from their equivalent widths are in very good agreement. However, we need two different values for $\xi_{\mathrm{m}}$ in a synthesis of their profiles.

Analyzing these different values for $\xi_{\mathrm{m}}$, we found a surprisingly tight correlation of the macroturbulent velocity with the (reduced) equivalent width (classical correlation coefficient $\rho=0.81$ ). A simple least squares fit gives

$\xi_{\mathrm{m}}=10.9 \log \left(W_{\lambda} / \lambda\right)+71.7$

Further inspection of the results gives an offset of $\sim 2.3 \mathrm{~km} \mathrm{~s}^{-1}$ between lines of neutral and lines of ionised species with the same reduced width. Therefore, we can specify the previous relation by making the distinction between lines of neutral and lines of ionised species:

$\xi_{\mathrm{m}}($ neutral lines $)=10.9 \log \left(W_{\lambda} / \lambda\right)+70.2 \pm 2.7$

$\xi_{\mathrm{m}}($ ionised lines $)=10.9 \log \left(W_{\lambda} / \lambda\right)+72.4 \pm 3.2$ 


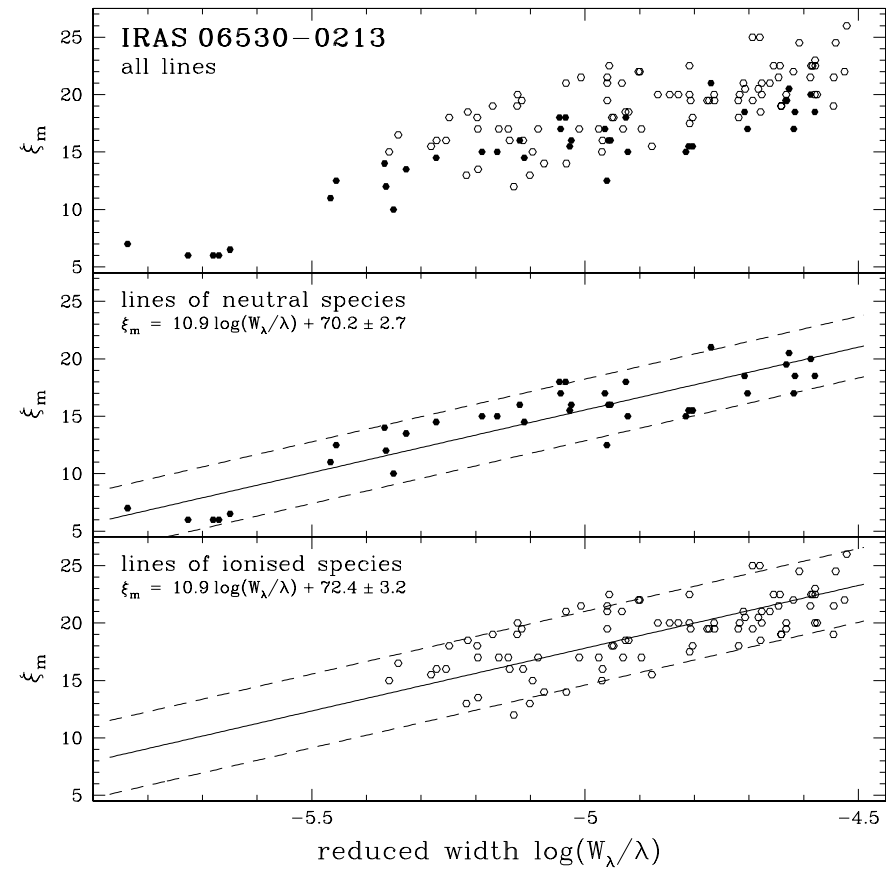

Fig. 4. Relation between the (reduced) equivalent width $\log \left(W_{\lambda} / \lambda\right)$ and the macroturbulent velocity $\xi_{\mathrm{m}}$ of 121 lines used in the analysis of IRAS 06530-0213. The lines from ionised species have systematically higher macroturbulent velocities than lines from neutral species of the same width (upper panel). Therefore, two separate relations are deduced (middle and lower panel). The errorbars are the standard deviations on the mean trend multiplied by 1.5 .

where the errorbar is empirically deduced from Fig. 4 (being 1.5 times the standard deviation on the mean trend). The correlation coefficient for lines of neutral species is $\rho_{\mathrm{n}}=0.90$; the same coefficient for lines of singly ionised species is $\rho_{\mathrm{i}}=0.70$. The reason for this dependence is not clear, nor a description of this effect was found in literature. This might be due to the fact that most authors deduce the macroturbulent velocity from just a few lines or lines in the immediate proximity of the line/region under interest. We interpret this effect as an optical depth effect of the macroturbulent velocity, the stronger lines being formed on average at lower geometrical depth. We want to stress that a similar relation, however less documented, was found for HD 172481 (Reyniers \& Van Winckel 2001), so it is certainly not an isolated peculiarity of the spectrum of IRAS 06530-0213 studied here.

\subsubsection{O synthesis}

Due to the high excitation potential of the oxygen lines, a reliable oxygen abundance is always difficult to derive in this temperature-gravity domain, and mostly only the oxygen triplet at $6155 \AA$ is accessible. The situation is even worse for heavily s-enriched objects like IRAS 06530-0213 since the triplet is significantly blended by lines of s-process species. Important blends at the position of the triplet are lines of Fe I (6157.73 $\AA$ ), Pr II (6157.81 $\AA$ ) and Nd II (6157.82 $\AA$ ). Therefore, the only way to obtain a reliable oxygen abundance in heavily enriched objects is by spectrum synthesis. For the $\mathrm{O}$ lines themselves, we used the atomic data published by Biémont et al. (1991).
Table 5. Results of the spectrum synthesis of the OI triplet of IRAS 06530-0213.

\begin{tabular}{ccccc}
\hline \hline $\begin{array}{c}\lambda \\
(\AA)\end{array}$ & $\begin{array}{c}\chi \\
(\mathrm{eV})\end{array}$ & $\log g f$ & $\begin{array}{c}\text { adopted } \xi_{\mathrm{m}} \\
\left(\mathrm{km} \mathrm{s}^{-1}\right)\end{array}$ & $\log \epsilon$ \\
\hline 6155.971 & 10.74 & -0.674 & $13.5( \pm 3)$ & $8.58( \pm 0.06)$ \\
6156.778 & 10.74 & -0.453 & $15.25( \pm 3)$ & $8.71( \pm 0.09)$ \\
6158.187 & 10.74 & -0.307 & $16.25( \pm 3)$ & $8.62( \pm 0.05)$ \\
\cline { 4 - 5 } & & & & 8.64 \\
\hline
\end{tabular}

Furthermore, the relation between equivalent width and macroturbulent velocity presented above causes a double difficulty when making the synthesis. First, the relation is deduced for single, non-blended lines. Hence, it is not clear how the relation should be applied to blended lines. Second, since the three lines are of different strength, each line requires its own macroturbulent broadening, so that the lines of the triplet had to be separately fitted. As a consequence, it is also difficult to show the synthesis on one single figure. Therefore, the results are summarized in a table (Table 5).

\subsection{Elements beyond the Ba peak}

Inspired by the detection of a hafnium (Hf, $Z=72)$ line in the objects IRAS 07134+1005 and IRAS 19500-1709 as reported in Paper I, we initiated a systematic search for other elements beyond the Ba peak. Our VLT+UVES spectra of the heavily enriched objects are ideally suited for this search since they combine a large wavelength coverage with a high signal-tonoise. Moreover, the release of a new database with atomic data of the lanthanides $(Z=57-71)$ at Mons University (B), the so called DREAM project, is an invaluable supplement to the VALD database which was our only source of atomic data of species in this mass range till now. The potential of this new database was recently illustrated by Reyniers et al. (2002). Since it also contains atomic data for doubly ionised lanthanides, we extended our search to these ions. The details of our successful search are published in a dedicated letter (Reyniers \& Van Winckel 2003), while here we only report on the results of the two programme stars. We have derived abundances of gadolinium ( $\mathrm{Gd}, Z=64)$, ytterbium (Yb, $Z=$ 70), lutetium ( $\mathrm{Lu}, Z=71$ ) and tungsten (W, $Z=74$ ) for IRAS 06530-0213, and of Gd and Lu for IRAS 08143-4406.

\subsection{Hyperfine structure}

In Paper I we studied the influence of hyperfine splitting (hfs) on the abundances of elements which are considered to be sensitive for this effect. The general conclusion of this exercise was that the hfs effect on the lines used in the abundance analysis is very small, due to the relatively high effective temperature of the $21 \mu \mathrm{m}$ stars. This is only true for weak lines, while for stronger lines an effect is indeed expected. Such strong lines were, however, not used in the abundance determination. Because several papers discussing hfs constants for different elements recently appeared in literature, we decided to repeat this exercise for the two program stars in this paper. The influence 
of hfs was determined by calculating the equivalent width of a studied line, with and without hfs decomposition. In this way, the exact profile of the line is eliminated, and no broadening factors have to be applied. We focussed on the s-process elements La, Eu and Lu.

For La, hfs constants A and B were taken from Lawler et al. (2001a). The La line list of IRAS 06530-0213 contains only one line with hfs constants for both levels $(5482.268 \AA$, $W_{\lambda}=91.8 \mathrm{~m} \AA$ ). The effect of hfs on the abundance is smaller than 0.01 dex. The La line list of IRAS 08143-4406 contains eight lines for which there are hfs constants in Lawler et al. (2001a). For seven of these lines, the effect is negligible $(<0.02 \mathrm{dex})$, while for the strongest line in the list $(6262.287 \AA$, $W_{\lambda}=148.0 \mathrm{m \AA}$ ), the effect is significant. An hfs-treatment of this line yields an abundance of $2.60 \mathrm{dex}$, which is $0.15 \mathrm{dex}$ lower than a treatment without hfs.

The Eu abundance is often considered to be strongly hfs sensitive, and an hfs treatment is indispensable when studying Eu abundances in cool stars. We studied the strongest Eu line of the two stars that was used in our analysis $(6645.064 \AA$, 103.2 $\mathrm{mA}$ in IRAS 06530-0213). A and B constants for this line were taken from Lawler et al. (2001b). The solar isotopic composition was applied. It turned out that, even for this relatively strong line with a strong decomposition, the effect on the derived abundance is marginal $(<0.02 \mathrm{dex})$. We also studied the effect of hfs on the Eu abundance of the $21 \mu \mathrm{m}$ stars of Paper I. Only for the cooler and strongly enriched IRAS $05341+0852$, the effect was noticeable (an abundance decrease of $0.05 \mathrm{dex}$ and $0.07 \mathrm{dex}$ for the lines at $6437.640 \AA$ and $7194.830 \AA$ respectively).

While for $\mathrm{La}$ and $\mathrm{Eu}$, the effect of hfs on the abundance is much smaller than other uncertainties $(\log g f$, continuum placement, undetected blends, ... ), the situation is different for the Lu abundance. Hfs constants for the two detected Lu lines were taken from Brix \& Kopfermann (1952) and Den Hartog et al. (1998). In the case of IRAS 06530-0213, the difference in abundance between a hfs and a non-hfs synthesis is $-0.27 \mathrm{dex}$ for the $6221.890 \AA$ line (see Fig. 5), and -0.14 dex for the $6463.107 \AA$ line. For the less s-enhanced IRAS 08143-4406, hfs only affects the line profile of the two Lu lines, but not their equivalent width.

\subsection{Abundance results}

In Table 6 we present the complete abundance analysis results of IRAS 06530-0213 and IRAS 08143-4406. The same results are graphically presented in Fig. 6. In this figure, the different groups of elements are marked with different symbols. We will now summarize the main results for each of these groups.

Metallicity Both stars are (moderately) metal deficient, with iron abundances of $[\mathrm{Fe} / \mathrm{H}]=-0.5$ and -0.4 for IRAS 06530-0213 and IRAS 08143-4406 respectively. The other iron peak elements all follow this deficiency, although zinc $(\mathrm{Zn})$ is somewhat higher than expected for IRAS 08143-4406.

CNO-elements Both stars are clearly carbon enriched, with a huge enhancement in the case of IRAS 06530-0213

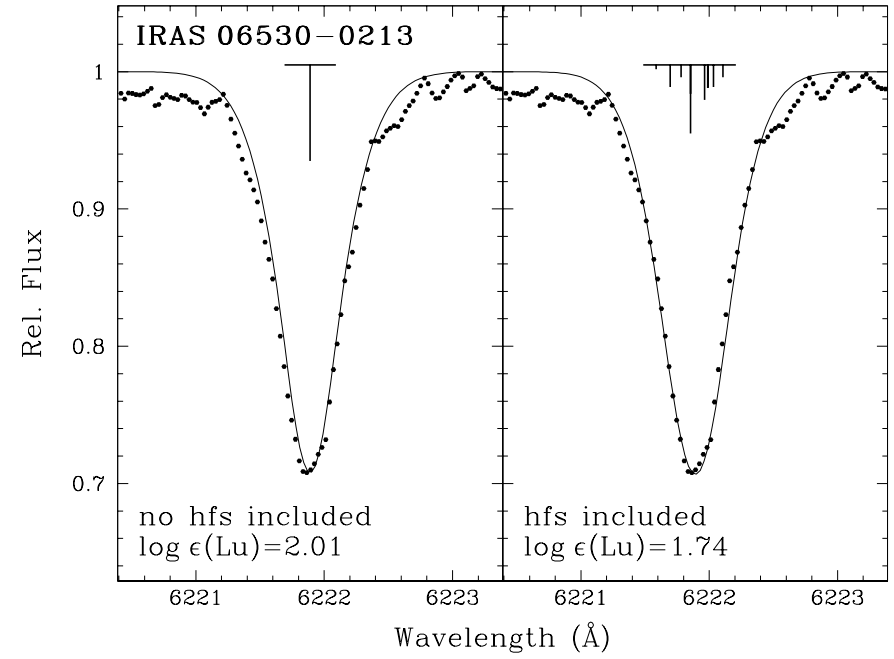

Fig. 5. Synthesis of the $6221.890 \AA$ Lu line in IRAS 06530-0213, without (left panel) and with (right panel) hyperfine decomposition. The adopted abundance is indicated below. The difference in abundance illustrates the necessity of the inclusion of hfs for this line.

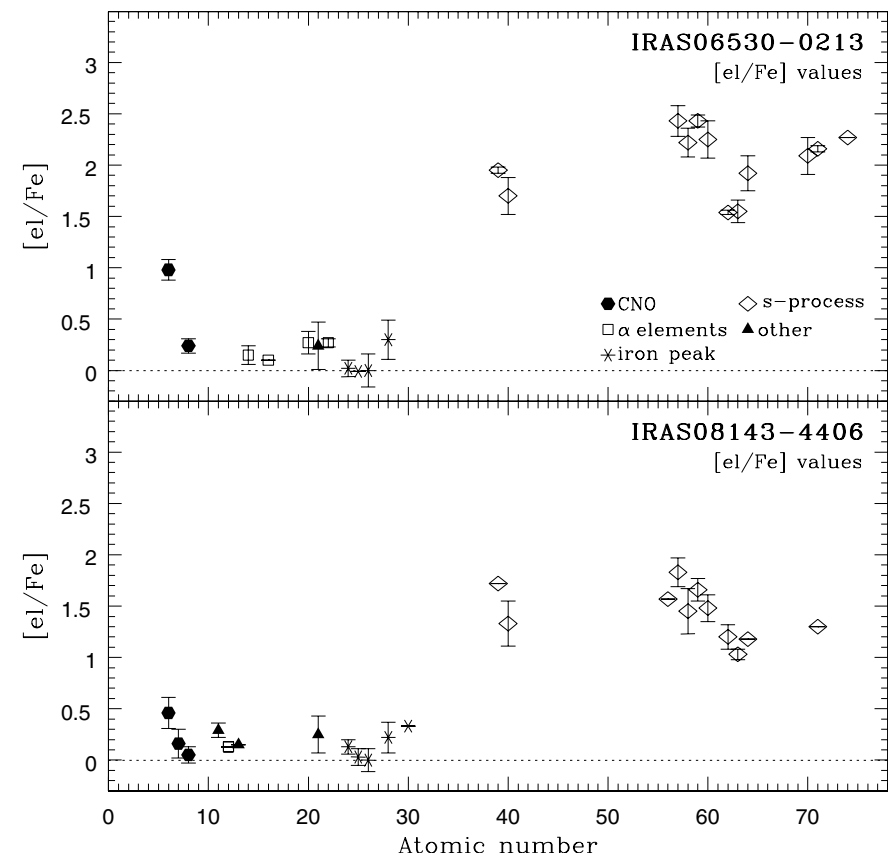

Fig. 6. [el/Fe] values for IRAS 06530-0213 and IRAS 08143-4406. The errorbars are the line-to-line scatters; the dotted line represents $[\mathrm{el} / \mathrm{Fe}]=0$. It is already clear from the figure that a higher neutron irradiation is expected for IRAS 06530-0213.

$([\mathrm{C} / \mathrm{Fe}]=+1.0)$. As a consequence, we derive a high $\mathrm{C} / \mathrm{O}$ number ratio of $\mathrm{C} / \mathrm{O}=2.8$ for this star; For IRAS 08143-4406, this ratio is 1.3 . One has to note, however, that the errors on mainly the oxygen abundance preclude an accurate $\mathrm{C} / \mathrm{O}$ number ratio determination based on photospheric atomic lines.

$\alpha$-elements The simple mean of the $[\mathrm{el} / \mathrm{Fe}]$ values of the (available) $\alpha$-elements $\mathrm{Mg}, \mathrm{Si}, \mathrm{S}, \mathrm{Ca}$ and Ti yields for both stars $[\alpha / \mathrm{Fe}]=+0.2$. Such an enhancement is normal for stars in this metallicity range, as a consequence of the galactic chemical evolution and therefore does not correspond to an intrinsic enhancement. 
Table 6. Abundance results for the two programme stars. Because of a time gap of 16 days between the DI2 and RED580 UVES setting for IRAS 08143-4406, we had to adopt 2 different sets of atmospheric parameters. The parameters for the RED580 setting are deduced using the iron lines. The parameters for the DI2 setting are deduced relative to the parameters of the RED580 setting, by a detailed study of 26 lines in the overlapping region. The following solar abundances are adopted: for the solar iron abundance we used the meteoritic iron abundance of 7.51; the references for the solar CNO are C: Biémont et al. (1993), N: Hibbert et al. (1991) and O: Biémont et al. (1991); for Mg and Si the latest Holweger values (Holweger 2001) were used; for La and Eu we took the recent values derived by Lawler et al. (2001a) and Lawler et al. (2001b) respectively; other solar abundances were taken from the review by Grevesse \& Sauval (1998). Despite the fact that there are more recent values for some of the solar abundances (especially for the solar $\mathrm{CNO}$ ), we take these references to ensure as much as possible the $g f$ values that we have used in the present paper to be consistent with the adopted solar abundances.

\begin{tabular}{|c|c|c|c|c|c|c|c|c|c|c|c|c|c|c|c|c|c|}
\hline \multirow[b]{2}{*}{ ion } & \multicolumn{5}{|c|}{$\begin{array}{c}\text { IRAS 06530-0213 } \\
{[\mathrm{Fe} / \mathrm{H}]=-0.46}\end{array}$} & \multicolumn{3}{|c|}{$\begin{array}{l}\text { RED580 setting } \\
\begin{aligned} &(2001 / 02 / 01) \\
& T_{\text {eff }}=7250 \mathrm{~K} \\
& \log g=1.5(\mathrm{cgs}) \\
& \xi_{\mathrm{t}}=5.5 \mathrm{~km} \mathrm{~s}^{-1}\end{aligned}\end{array}$} & \multicolumn{3}{|c|}{$\begin{aligned} \text { DI2 setting } \\
(2001 / 01 / 16) \\
T_{\text {eff }}=7050 \mathrm{~K} \\
\log g=1.2\left(\mathrm{cgs}^{2}\right) \\
\xi_{\mathrm{t}}=5.5 \mathrm{~km} \mathrm{~s}^{-1}\end{aligned}$} & \multicolumn{5}{|c|}{ final result } & \\
\hline & $N$ & $\overline{W_{\lambda}}$ & $\log \epsilon$ & $\sigma$ & {$[\mathrm{el} / \mathrm{Fe}]$} & $N$ & $\log \epsilon$ & $\sigma$ & $N$ & $\log \epsilon$ & $\sigma$ & $N$ & $\overline{W_{\lambda}}$ & $\log \epsilon$ & $\sigma$ & {$[\mathrm{el} / \mathrm{Fe}]$} & $\log \epsilon_{\odot}$ \\
\hline$\overline{C I}$ & 8 & 79 & 9.09 & 0.10 & 0.98 & 13 & 8.66 & 0.15 & 11 & 8.64 & 0.14 & 23 & 64 & 8.64 & 0.15 & 0.46 & 8.57 \\
\hline N I & & & & & & & & & 7 & 7.76 & 0.14 & 7 & 62 & 7.76 & 0.14 & 0.16 & 7.99 \\
\hline OI & 3 & $s s$ & 8.64 & 0.07 & 0.24 & 5 & 8.52 & 0.08 & & & & 5 & 34 & 8.52 & 0.08 & 0.05 & 8.86 \\
\hline $\mathrm{NaI}$ & & & & & & 4 & 6.23 & 0.07 & & & & 4 & 39 & 6.23 & 0.07 & 0.29 & 6.33 \\
\hline Mg I & & & & & & 1 & 7.28 & & & & & 1 & 40 & 7.28 & & 0.13 & 7.54 \\
\hline $\mathrm{Al} \mathrm{I}$ & & & & & & 1 & 6.38 & & 2 & 6.22 & & 2 & 10 & 6.23 & & 0.15 & 6.47 \\
\hline Si I & 1 & 43 & 7.29 & & 0.21 & 11 & 7.58 & 0.16 & 3 & 7.46 & 0.15 & 13 & 28 & 7.57 & 0.15 & 0.42 & 7.54 \\
\hline Si II & 1 & 85 & 7.16 & & 0.08 & & & & & & & & & & & & 7.54 \\
\hline S I & 1 & 44 & 6.97 & & 0.10 & 3 & 7.12 & 0.01 & 6 & 7.07 & 0.11 & 6 & 55 & 7.07 & 0.11 & 0.13 & 7.33 \\
\hline $\mathrm{Ca} \mathrm{I}$ & 6 & 67 & 6.17 & 0.11 & 0.27 & 12 & 6.10 & 0.17 & & & & 12 & 60 & 6.10 & 0.17 & 0.13 & 6.36 \\
\hline Ca II & & & & & & 1 & 5.91 & & & & & 1 & 16 & 5.91 & & -0.06 & 6.36 \\
\hline Sc II & 3 & 82 & 2.95 & 0.23 & 0.24 & 4 & 3.03 & 0.18 & & & & 4 & 70 & 3.03 & 0.18 & 0.25 & 3.17 \\
\hline Ti II & 3 & 99 & 4.83 & 0.04 & 0.27 & 2 & 5.05 & & & & & 2 & 127 & 5.05 & & 0.42 & 5.02 \\
\hline $\mathrm{Cr} \mathrm{I}$ & 1 & 18 & 5.27 & & 0.06 & 3 & 5.47 & 0.06 & 3 & 5.44 & 0.07 & 6 & 53 & 5.45 & 0.06 & 0.17 & 5.67 \\
\hline Cr II & 9 & 78 & 5.22 & 0.08 & 0.01 & 9 & 5.40 & 0.09 & 1 & 5.33 & & 9 & 86 & 5.39 & 0.09 & 0.11 & 5.67 \\
\hline Mn I & 1 & 14 & 4.92 & & -0.01 & 2 & 5.03 & & 2 & 5.06 & & 3 & 59 & 5.03 & 0.08 & 0.03 & 5.39 \\
\hline $\mathrm{Fe} \mathrm{I}$ & 22 & 70 & 7.02 & 0.13 & -0.03 & 48 & 7.11 & 0.12 & 3 & 7.16 & 0.05 & 49 & 59 & 7.12 & 0.12 & & 7.51 \\
\hline $\mathrm{Fe}$ II & 7 & 83 & 7.05 & 0.16 & & 6 & 7.14 & 0.09 & 1 & 6.95 & & 7 & 83 & 7.12 & 0.11 & & 7.51 \\
\hline $\mathrm{Ni}$ I & 4 & 22 & 6.09 & 0.19 & 0.30 & 13 & 6.10 & 0.17 & 10 & 6.07 & 0.12 & 19 & 41 & 6.08 & 0.15 & 0.22 & 6.25 \\
\hline $\mathrm{Zn} \mathrm{I}$ & & & & & & 1 & 4.51 & & 2 & 4.53 & & 2 & 82 & 4.54 & & 0.33 & 4.60 \\
\hline $\mathrm{Y}_{\text {II }}$ & 2 & $b l$ & 3.73 & 0.03 & 1.95 & 1 & 3.58 & & 1 & 3.55 & & 2 & 119 & 3.57 & & 1.72 & 2.24 \\
\hline Zr II & 6 & 81 & 3.84 & 0.18 & 1.70 & 6 & 3.57 & 0.26 & 4 & 3.54 & 0.12 & 8 & 59 & 3.54 & 0.22 & 1.33 & 2.60 \\
\hline Ba II & & & & & & & & & 1 & 3.31 & & 1 & 33 & 3.31 & & 1.57 & 2.13 \\
\hline La II & 13 & 77 & 3.10 & 0.15 & 2.43 & 28 & 2.59 & 0.16 & 13 & 2.55 & 0.09 & 37 & 62 & 2.57 & 0.14 & 1.83 & 1.13 \\
\hline Ce II & 18 & 74 & 3.34 & 0.14 & 2.22 & 14 & 2.59 & 0.21 & 10 & 2.70 & 0.24 & 24 & 55 & 2.64 & 0.22 & 1.45 & 1.58 \\
\hline Pr II & 8 & 84 & 2.68 & 0.06 & 2.43 & 9 & 1.98 & 0.11 & & & & 9 & 40 & 1.98 & 0.11 & 1.66 & 0.71 \\
\hline Pr III & 2 & 53 & 2.54 & 0.30 & 2.29 & & & & & & & & & & & & 0.71 \\
\hline Nd II & 24 & 91 & 3.29 & 0.18 & 2.25 & 27 & 2.60 & 0.13 & 9 & 2.64 & 0.17 & 33 & 58 & 2.59 & 0.13 & 1.48 & 1.50 \\
\hline Nd III & 2 & 124 & 3.05 & 0.01 & 2.01 & & & & & & & & & & & & 1.50 \\
\hline Sm II & 2 & 34 & 2.09 & 0.02 & 1.54 & 3 & 1.88 & 0.16 & 17 & 1.81 & 0.12 & 18 & 61 & 1.82 & 0.12 & 1.20 & 1.01 \\
\hline Eu II & 2 & 72 & 1.61 & 0.11 & 1.55 & 2 & 1.18 & & 1 & 1.14 & & 3 & 32 & 1.16 & 0.05 & 1.03 & 0.52 \\
\hline Gd II & 3 & 41 & 2.58 & 0.17 & 1.92 & 2 & 1.91 & & & & & 2 & 10 & 1.91 & & 1.18 & 1.12 \\
\hline Yb II & 2 & 47 & 2.71 & 0.18 & 2.09 & & & & & & & & & & & & 1.08 \\
\hline Lu II & 2 & ss & 1.76 & 0.03 & 2.16 & 1 & 0.97 & & & & & 1 & 42 & 0.97 & & 1.30 & 0.06 \\
\hline W II & 1 & $b l$ & 2.92 & & 2.27 & & & & & & & & & & & & 1.11 \\
\hline
\end{tabular}

ss: spectrum synthesis; $b l$ : line is blended, but abundance is corrected for this blend, using a dedicated MOOG routine

s-process elements From Fig. 6, it is clear that the s-process enrichment of the two objects under study is very strong. This enrichment is the most important argument for the post third dredge-up status of the two stars.
The s-process elements observed in evolved stars can be divided into two groups: the light s-process elements around the magic neutron number $50(\mathrm{Sr}, \mathrm{Y}, \mathrm{Zr})$ and the heavy s-process elements around the magic neutron number 82 (Ba, La, Ce, Pr, 
$\mathrm{Nd}, \mathrm{Sm})$. In order to study the s-process pattern in more detail, four indices are generally defined: $[\mathrm{s} / \mathrm{Fe}],[\mathrm{ls} / \mathrm{Fe}],[\mathrm{hs} / \mathrm{Fe}]$ and [hs/ls]. Which elements are taken into account to determine these indices is different from author to author and is mainly determined by the possibility to compute accurate abundances of the different elements. To be able to compare our results with our results on the $21 \mu \mathrm{m}$ objects (Paper I), we define the ls-index as the mean of $\mathrm{Y}$ and $\mathrm{Zr}$ and the hs-index as the mean of $\mathrm{Ba}, \mathrm{La}, \mathrm{Nd}$ and $\mathrm{Sm}$. Consequently, $[\mathrm{s} / \mathrm{Fe}]$ is the mean of the former six elements and $[\mathrm{hs} / \mathrm{ls}]=[\mathrm{hs} / \mathrm{Fe}]-[\mathrm{ls} / \mathrm{Fe}]$.

With the definitions from above, the s-process indices for IRAS 06530-0213 are: $[\mathrm{s} / \mathrm{Fe}]=+2.1$, $[1 \mathrm{~s} / \mathrm{Fe}]=+1.8$, $[\mathrm{hs} / \mathrm{Fe}]=+2.2$ and $[\mathrm{hs} / \mathrm{ls}]=+0.4$; for IRAS 08143-4406, they are: $[\mathrm{s} / \mathrm{Fe}]=+1.5,[1 \mathrm{~s} / \mathrm{Fe}]=+1.5,[\mathrm{hs} / \mathrm{Fe}]=$ +1.5 and $[\mathrm{hs} / \mathrm{ls}]=0.0$. Note that the $\mathrm{Ba}$ abundance for IRAS 06530-0213 had to be estimated in order to calculate the $[\mathrm{hs} / \mathrm{Fe}]$ and $[\mathrm{hs} / \mathrm{ls}]$ indices. We estimated this abundance in the same way as explained in Paper I, by using the tables of Malaney (1987) and an exponential distribution of neutron exposures of $\tau_{0}=0.4 \mathrm{mbar}^{-1}$. The Ba abundance estimated from the $\mathrm{La}$ abundance is then $[\mathrm{Ba} / \mathrm{Fe}]=+2.55$.

These abundance analyses make IRAS 06530-0213 and IRAS 08143-4406 join the group of post-AGB stars that clearly display chemical evidence for a third dredge-up event. They share the same chemical signatures as the $21 \mu \mathrm{m}$ objects discussed in Paper I. IRAS 06530-0213 is s-process enhanced at approximately the same level as IRAS $05341+0852$, the most s-process enriched intrinsic object found so far.

\subsection{Comparison with Hrivnak \& Reddy (2003)}

An independent abundance analysis of IRAS 06530-0213 has recently been carried out by Hrivnak \& Reddy (2003), based on high-resolution spectra taken with the $2.7 \mathrm{~m}$ telescope at McDonald Observatory. The authors derived a slightly lower temperature of $6900 \mathrm{~K}$, resulting in a lower metallicity of $[\mathrm{Fe} / \mathrm{H}]=-0.9$. The abundances relative to iron of the elements that are in common in both analyses are, however, in good agreement. One should note that the analysis presented here is based on spectra with both a higher resolution (56000 vs. 45000$)$ and a higher signal-to-noise (100 vs. 30$)$. As a consequence, our analysis does not only include more lines (149 vs. 102), it also covers much more ions (28 vs. 17).

\section{Neutron exposure}

In Paper I (Sect. 6) we compared the strength of the neutron irradiation parameterized by the $[\mathrm{hs} / \mathrm{ls}]$ index with both the total s-process enrichment and the metallicity for the $21 \mu \mathrm{m}$ stars. A surprising result from this comparison was the strong correlation between the $[\mathrm{hs} / \mathrm{ls}]$ index and the $[\mathrm{s} / \mathrm{Fe}]$ index implying an increase in efficiency of the neutron nucleosynthesis with increasing third dredge-up efficiency. This relation was, however, hampered by the low number statistics on which it was based: only six stars. Here we have two more data points (Table 7), one of which having a very strong enrichment (IRAS 06530-0213).

From Fig. 7 it is clear that the results of the two newly discovered objects strengthen the conclusions of Paper I. In the
Table 7. The metallicity and the s-process indices of the eight stars plotted in Fig. 7. Some values given in this table slightly differ from the values given in Paper I, due to minor revisions and updates that are described in detail in Reyniers (2002).

\begin{tabular}{cccccr}
\hline \hline object & {$[\mathrm{Fe} / \mathrm{H}]$} & {$[\mathrm{s} / \mathrm{Fe}]$} & {$[\mathrm{ls} / \mathrm{Fe}]$} & {$[\mathrm{hs} / \mathrm{Fe}]$} & {$[\mathrm{hs} / \mathrm{ls}]$} \\
\hline IRAS 04296+3429 & -0.6 & 1.5 & 1.7 & 1.5 & -0.2 \\
IRAS 05341+0852 & -0.7 & 2.2 & 2.0 & 2.3 & 0.3 \\
IRAS 06530-0213 & -0.5 & 2.1 & 1.8 & 2.2 & 0.4 \\
IRAS 07134+1005 & -1.0 & 1.5 & 1.6 & 1.5 & -0.1 \\
IRAS 08143-4406 & -0.4 & 1.5 & 1.5 & 1.5 & 0.0 \\
IRAS 19500-1709 & -0.7 & 1.2 & 1.4 & 1.0 & -0.4 \\
IRAS 22223+4327 & -0.3 & 1.1 & 1.4 & 0.9 & -0.4 \\
IRAS 23304+6147 & -0.8 & 1.6 & 1.5 & 1.6 & 0.1 \\
\hline
\end{tabular}
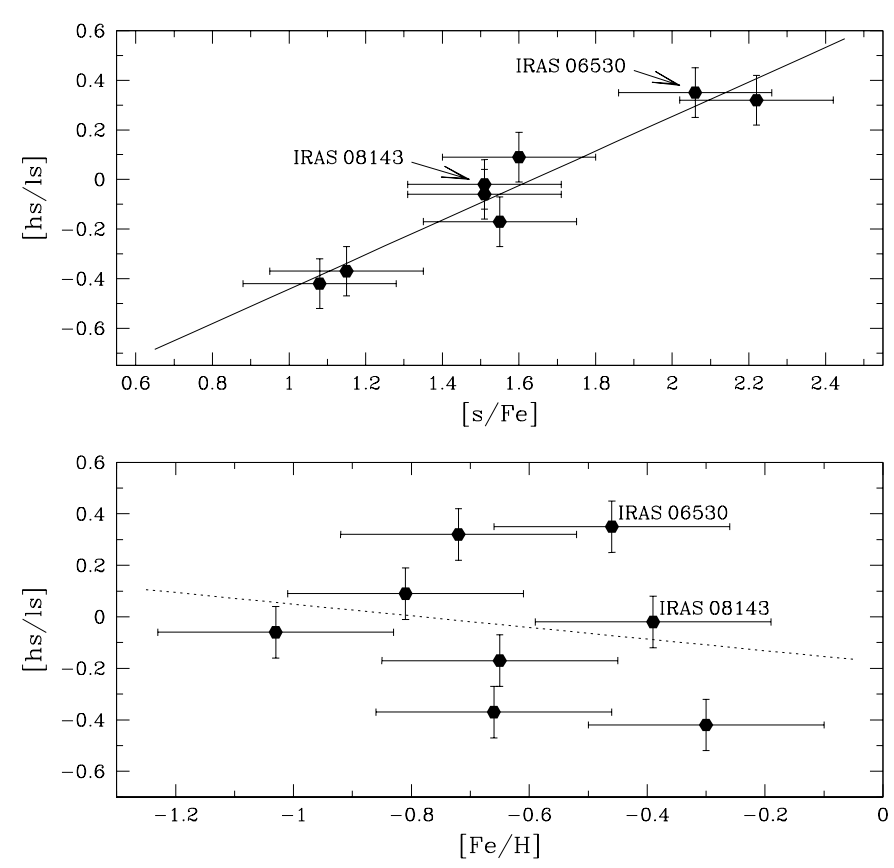

Fig. 7. Comparison of IRAS 06530-0213 and IRAS 08143-4406 with the $21 \mu \mathrm{m}$ stars of Paper I. The values of the data points in this figure are given in Table 7. Upper panel: The correlation between the total enrichment of s-process elements ( $[\mathrm{s} / \mathrm{Fe}]$, which is the mean of $[\mathrm{Y} / \mathrm{Fe}],[\mathrm{Zr} / \mathrm{Fe}],[\mathrm{Ba} / \mathrm{Fe}],[\mathrm{La} / \mathrm{Fe}],[\mathrm{Nd} / \mathrm{Fe}]$ and $[\mathrm{Sm} / \mathrm{Fe}])$ and the [hs/ls] index. The straight line gives the simple least-squares fit. Lower panel: The $[\mathrm{hs} / \mathrm{ls}]$ index as a function of the metallicity determined by the Fe abundance. The dotted line gives the simple least-squares fit $[\mathrm{hs} / \mathrm{ls}]=-0.23[\mathrm{Fe} / \mathrm{H}]-0.18$.

upper panel of this figure, the two data points for the newly discovered enriched stars nicely fit the correlation between the [hs/ls] index and the $[\mathrm{s} / \mathrm{Fe}]$ index. The new correlation coefficient for the eight stars is +0.96 , the same as found in Paper I, while a least-squares fit gives $[\mathrm{hs} / \mathrm{ls}]=0.70[\mathrm{~s} / \mathrm{Fe}]-1.14$. Especially the analysis of IRAS 06530-0213 yields an important data point to extend the relation towards stronger enrichments. In turn, the addition of the new objects dramatically illustrates the weak correlation between the metallicity and the [hs/ls] index (lower panel of Fig. 7). Whereas the correlation coefficient for these two quantities for the $21 \mu \mathrm{m}$ stars was still -0.55 , it decreases to -0.19 after addition of the two new objects. Mainly the strong enrichment of IRAS 06530-0213 compared to its only mild metal deficiency is a strong indication to 


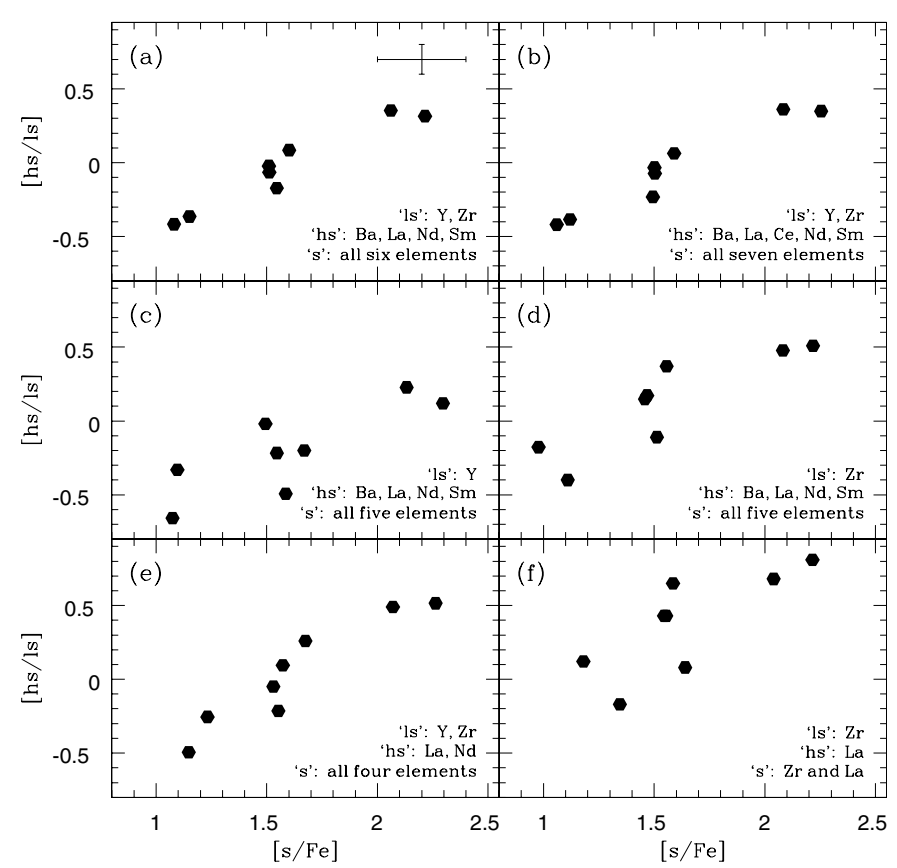

Fig. 8. Study of the dependence of the proposed relation between the $[\mathrm{s} / \mathrm{Fe}]$ index and $[\mathrm{hs} / \mathrm{ls}]$ index upon the particular definition of the indices. Despite the scatter on the mean trend for some definitions, the relation holds for each definition presented here. Especially the definition proposed by Busso et al. (2001) (panel e) seems to be an excellent alternative for the "default" definition of panel a.

suspect a large intrinsic spread of integrated neutron irradiations (or, alternatively, ${ }^{13} \mathrm{C}$ pocket efficiencies). One can even doubt the presence of any relation between $[\mathrm{Fe} / \mathrm{H}]$ and $[\mathrm{hs} / \mathrm{ls}]$ after inspection of Fig. 7.

The correlation between $[\mathrm{hs} / \mathrm{ls}]$ and $[\mathrm{s} / \mathrm{Fe}]$ is obviously dependent on the specific choice of the elements involved in the calculation of both the $[\mathrm{s} / \mathrm{Fe}]$ and $[\mathrm{hs} / \mathrm{ls}]$ index. The choice to incorporate $\mathrm{Y}$ and $\mathrm{Zr}$ for the calculation of $[1 \mathrm{~s} / \mathrm{Fe}]$ and $\mathrm{Ba}, \mathrm{La}$, $\mathrm{Nd}$ and $\mathrm{Sm}$ for $[\mathrm{hs} / \mathrm{Fe}$ ] was based on the choice made by Busso et al. (1995). Some of these elements (like Ba and Sm) are, however, not always observed and abundances of unobserved elements were estimated using the Malaney (1987) tables (see Sect. 4.5). It is an interesting exercise to investigate a possible dependence of the relation presented above upon the specific elements used in the indices. In Fig. 8, the relation between the neutron irradiation and the total s-process enrichment is revisited with different elements for the indices.

On panel (a) of this figure, the relation is drawn for the "default" index definition. On panel (b), the Ce abundance is incorporated in the "hs" (and hence "s") definition, but the points hardly change their position on the graph. On the middle panels the "ls" index is defined by only one element, being $Y$ on panel (c) and $\mathrm{Zr}$ on panel (d). This change has a rather large impact on the position of the points. The restriction to one abundance for the definition of the "ls" index does not seem favourable in this context. Especially if the "ls" index is solely defined by the $Y$ abundance, the relation is clearly less well defined. In Sect. 6 we further focus on the problems with this particular abundance. On the two lower panels, the "hs" definition has been varied. On panel (e), the [hs/ls] definition is the same as in Busso et al. (2001). With this definition, only observed abundances are used, none were estimated. Moreover, the relation found in panel (a) is very well reproduced with this definition. Hence, restricting hs to $\mathrm{La}$ and $\mathrm{Nd}$, the definition seems to be a very good alternative for the "default" definition. In the last panel, panel (f), a "minimal" definition is given with only two elements, $\mathrm{Zr}$ and La, which makes the scatter on the mean trend to become quite high, but still a strong hint to the proposed relation exists.

\section{Comparison with AGB nucleosynthetic models}

\subsection{AGB stellar models}

Although there is general consensus that the s-process nucleosynthesis is triggered by the engulfment of a small amount of protons of the hydrogen-rich envelope into the top layers of the He intershell (e.g. Busso et al. 1999, and references therein), there is not yet a selfconsistent nucleosynthetic AGB model. Different evolutionary codes use different assumptions, not only for the partial mixing zone, but also for the third dredgeup efficiency with or without overshoot assumptions (see comparative study of Lugaro et al. 2003, and references therein). Rotational mixing has recently been analysed and a strong effect seems to be a decrease in the neutron irradiation since the major neutron poison $\left({ }^{14} \mathrm{~N}\right)$ is mixed into the ${ }^{13} \mathrm{C}$-rich layers (Langer et al. 1999; Herwig et al. 2003).

In this section we compare our observed s-process distributions with the results of nucleosynthetic AGB models (Gallino et al. 1998; Busso et al. 1999, 2001). The nucleosynthesis predictions are calculated by a postprocessing code grafted upon the FRANEC evolutionary models (Straniero et al. 1997, 2003) that span a grid of models with masses of 1.3 to $3.0 M_{\odot}$ and metallicities from solar to $1 / 20$ solar. In the models, an ad hoc ${ }^{13} \mathrm{C}$ profile is introduced which follows basically an exponential distribution (see Fig.1 of Gallino et al. 1998) and which is burnt in radiative conditions during the interpulse phase, at a temperature of $T \sim 0.9 \times 10^{8} \mathrm{~K}$. Although the physical origin of the ${ }^{13} \mathrm{C}$ pocket remains to be studied, the models are used here to estimate the strength of the ${ }^{13} \mathrm{C}$ pocket needed to explain the observed s-process abundances. The ${ }^{13} \mathrm{C}$ pocket choice labeled "Standard Case" (ST) corresponds to $4 \times 10^{-6} M_{\odot}$ of ${ }^{13} \mathrm{C}$ and it is named in this way because it has been shown to best reproduce the "main component" of the s-process in the solar system when applied to low mass AGB stars of $[\mathrm{Fe} / \mathrm{H}]=-0.3$ (Arlandini et al. 1999). Other choices of ${ }^{13} \mathrm{C}$ pockets for individual AGB stars are then obtained by multiplication or division of this standard ${ }^{13} \mathrm{C}$ amount. As a consequence, different s-process patterns can be obtained for a fixed metallicity and mass. A spread in [hs/ls] such as found in the lower panel of Fig. 7 is therefore naturally found in these models when varying the strength of the ${ }^{13} \mathrm{C}$ pocket.

The FRANEC models allow the third dredge-up mechanism to self-consistently occur after a limited number of pulses, using the Schwarzschild criterion and without invoking any extra-mixing. Other AGB models (e.g. Goriely \& Mowlavi 2000; Herwig et al. 2003) use different prescriptions. Note that in the FRANEC models the thermal pulses with third dredge-up stop prior to the end of the AGB evolution, when the 
envelope is still relatively massive and the superwind has yet to develop. The mass loss prescription is also a critical point in each AGB model code, and different groups use different approaches (see e.g. Blöcker 1999, and references therein). The models presented here have been obtained using the mass loss parameterisation by Reimers (1975), with the choice $\eta=0.3$ for the free parameter.

It is clear that there is not yet a full theoretical understanding of the AGB nucleosynthesis and third dredge-up phenomena, making the matching between the models and the observed s-process distributions to be interpreted with caution. We want to stress, however, that the s-process enriched post-AGB objects are ideally suited to confront observed s-process distributions with theoretically predicted abundances, since (i) no dilution has to be accounted for (as in the case of extrinsic AGB-stars) and (ii) these objects fully completed their evolution through the TP-AGB phase (contrary to genuine AGB stars from the M-MS-S-SC-C sequence).

\subsection{Model fits for the $21 \mu \mathrm{m}$ stars of Paper I}

We made an attempt to fit the s-process distribution of the $21 \mu \mathrm{m}$ stars discussed in Paper I with the latest AGB models. The abundances were re-calculated using the latest MOOG version (April 2002). The abundances of IRAS 05341+0852 were re-determined using new VLT+UVES spectra. Although this new analysis is based on superior spectra (see Fig. 1) compared to the WHT+UES spectra in Paper I, the new results are not drastically different from our previous analysis.

The model parameters were found in the following way. We restricted the models to an initial mass of $1.5 M_{\odot}$. For the metallicity of the model, we used the spectroscopically derived value. The ${ }^{13} \mathrm{C}$ pocket is then the only parameter that is left over. It was found by manually fitting the observed $\mathrm{Zr}$ and $\mathrm{La}$ abundance (which is in fact representative for the [hs/ls] index). For two stars in the sample (IRAS 19500-1709 and IRAS 23304+6147) no solution could be found. For these two stars, the best fit was obtained with a model of lower initial mass of $1.3 M_{\odot}$. The fits are presented in Fig. 9. The fit for IRAS 19500-1709 still shows quite large discrepancies for the heavier elements. The abundances of these elements are, however, derived from only a few lines (see Paper I, for details), and should therefore be considered with caution.

The fits are not unique, but it turned out that a free choice between the possible ${ }^{13} \mathrm{C}$ pockets was rather well constrained due to the fixed metallicity. On the other hand, the amount of thermal pulses can influence the choice of the ${ }^{13} \mathrm{C}$ pocket: e.g. Busso et al. (2001) propose a ${ }^{13} \mathrm{C}$ pocket "ST/3" with 10 thermal pulses for the same abundances of IRAS $07134+1005$ we present here, whereas we found "ST/5". However, note that the present AGB model predictions have been considerably improved since the Busso et al. paper.

Finally, a small remark is given on the niobium $(\mathrm{Nb}, Z=$ 41) abundance. Since these models are for intrinsic stars, ${ }^{93} \mathrm{Zr}$ with a half-life of $1.5 \times 10^{6} \mathrm{yr}$, has not yet decayed to $\mathrm{Nb}$. In extrinsic stars, the $\mathrm{Nb}$ abundance is expected to be at the same level as Zr.

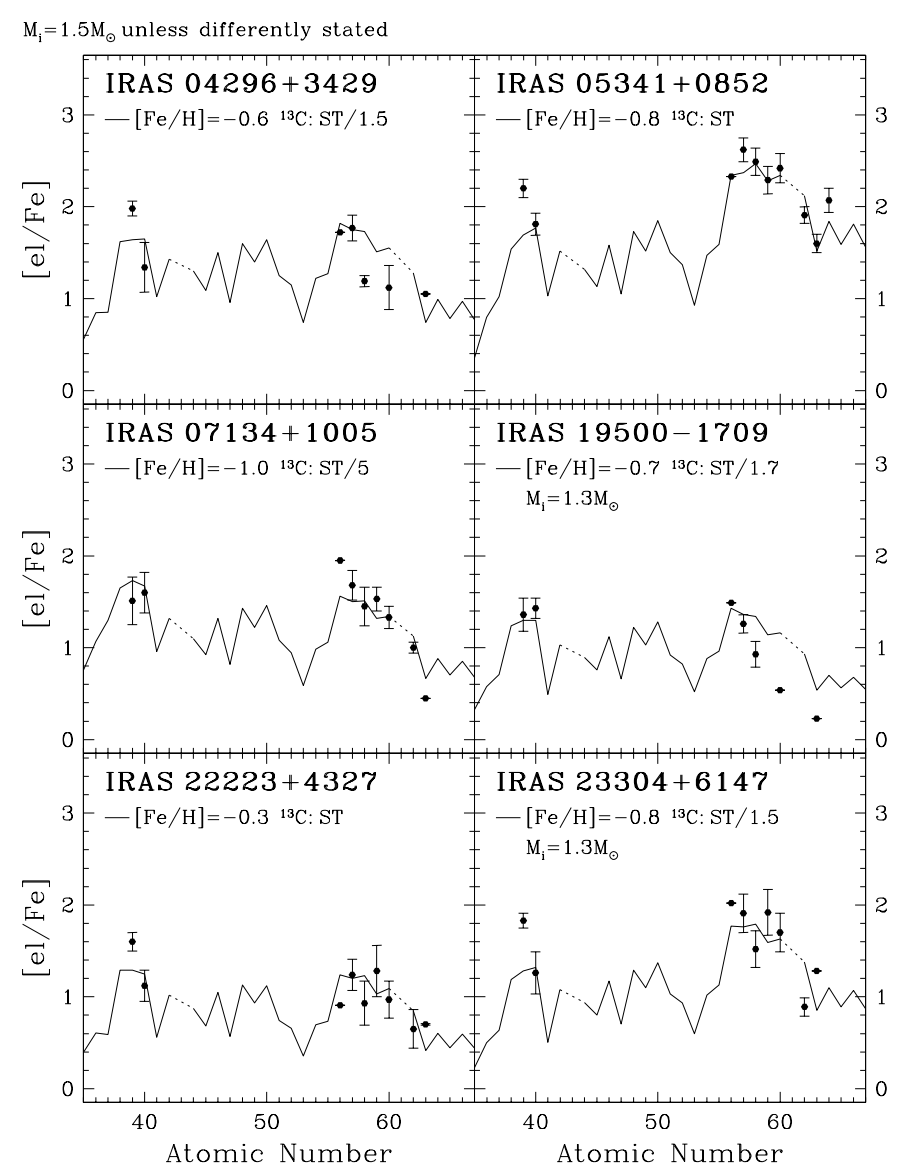

Fig. 9. Abundances of the s-process elements for the six $21 \mu \mathrm{m}$ stars of Paper I, together with their best fit AGB model. The model parameters are given in the upper left corner; the initial mass is taken $1.5 M_{\odot}$ for all models, except for IRAS 19500-1709 and IRAS 23304+6147. For these two stars, a lower initial mass of $1.3 M_{\odot}$ yielded a better fit. The errorbars plotted on the figure are the line-to-line scatters. The actual error on an abundance is, however, in most cases considerably larger than this value, due to uncertainties in the atmospheric parameters, undetected blends, etc. Note that in the AGB model predictions the solar meteoritic abundances by Anders \& Grevesse (1989) are used to convert the s-process yields to the actual $[\mathrm{el} / \mathrm{Fe}]$ predictions. This specific choice has only a marginal effect on the predictions in this elemental range. Choosing the solar abundances as listed in Table 6 to calculate the predictions, would introduce a maximum change of only $0.07 \mathrm{dex}(\mathrm{Pr})$ in the predictions.

\subsection{Model fits for the two programme stars}

After this first exercise, we searched for the best model fit for the two programme stars of this paper. The same fitting method was applied as for the $21 \mu \mathrm{m}$ stars, except that we extended the comparison to all observed elements. The final model fits, together with their parameters, are shown in Fig. 10. Note that decreasing the metallicity by e.g. $0.3 \mathrm{dex}$ (which corresponds to the metallicity found for IRAS $06530-0213$ by Hrivnak \& Reddy 2003) would result in a fit with a ${ }^{13} \mathrm{C}$ pocket which is decreased with the same factor, with respect to the original one.

\subsection{Model fit conclusions}

Apart from the fact that several solutions are possible for the same pattern, it is clear, though, that only one ${ }^{13} \mathrm{C}$ pocket 


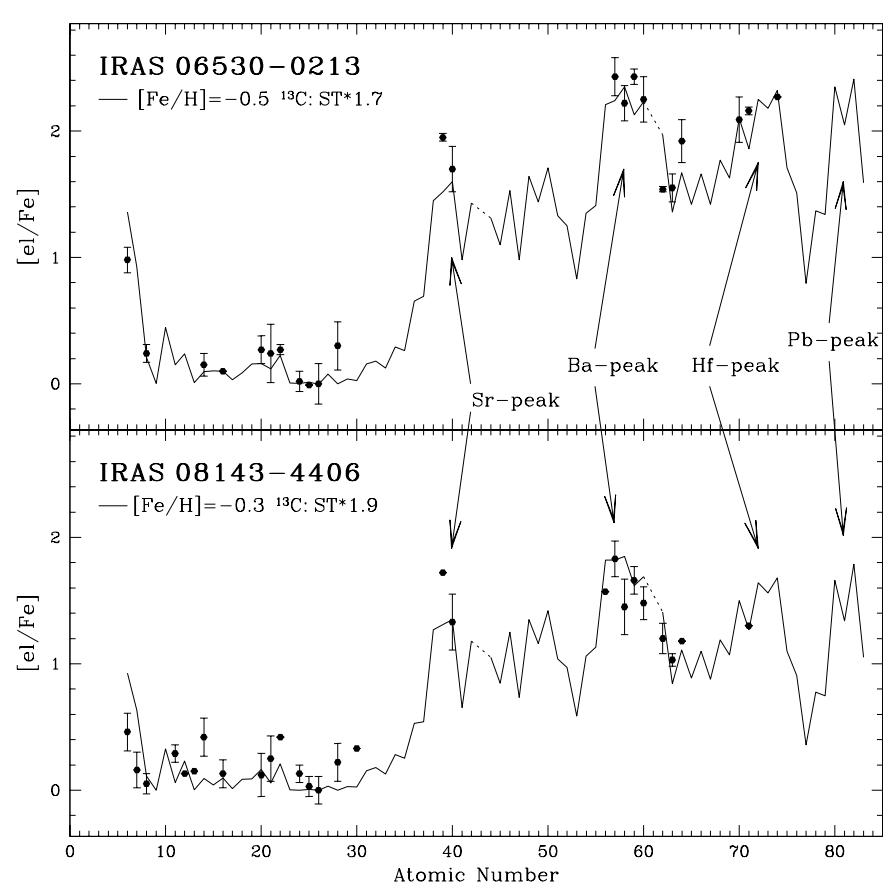

Fig. 10. Abundances of IRAS 06530-0213 and IRAS 08143-4406 together with their best fit AGB model. The model parameters are given in the upper left corner. The initial mass is $1.5 M_{\odot}$. Errorbars are the line-to-line scatters that are given in Table 6. Note that the use of the solar meteoritic abundances in the AGB model predictions (see also the caption of Fig. 9), introduces a rather large uncertainty for the $W$ abundance, for which the difference between solar photospheric and meteoritic abundance is very large $(0.43 \mathrm{dex}$, Anders \& Grevesse 1989).

alone cannot explain the abundance patterns in all eight objects. IRAS $07134+1005$ for example can certainly not be fitted with a ${ }^{13} \mathrm{C}$ pocket which exceeds "ST/2", whereas for IRAS $05341+0852$ a small pocket (<"ST") is not possible. This difference in ${ }^{13} \mathrm{C}$ pockets is much larger than the difference that could result from uncertainties in the models or in the abundances, and therefore we believe that the spread in ${ }^{13} \mathrm{C}$ pockets is real. The need of an intrinsic spread in the adopted ${ }^{13} \mathrm{C}$ pockets should be regarded as an essential result of this first exercise.

Other interesting conclusions can be drawn from the fits by inspecting the abundances individually. We give four examples to illustrate the mutual benefit of such fits for both the models and the observational analysis.

(1) The carbon abundance (not on the figure for the $21 \mu \mathrm{m}$ stars) is predicted far too high in each fit. Since this particular abundance is very reliable for each star $(\sigma<0.2)$, the too high prediction is probably a model's artefact.

(2) The yttrium abundance $(Z=39)$ is systematically $0.2 \mathrm{dex}$ too high for the cooler objects $\left(T_{\text {eff }} \lesssim 7000 \mathrm{~K}\right)$ in the sample when compared to the predicted abundances. For these cooler stars, only a few lines (typically $\sim 4$ ) were found since most Y II lines are too strong to deduce an accurate abundance. The two hotter stars in the sample (IRAS 07134+1005 and IRAS 19500-1709) do not show this effect, and since the hfs effect for $\mathrm{Y}$ is only very small, this discrepancy between the observed and the predicted abundance for the cooler stars is probably due to a $\log (g f)$ problem and/or blending of the Y II-lines used in these stars.

(3) Another, although minor, discrepancy is that the cerium abundance is systematically too low compared to the predictions for at least five objects. In this context it is noteworthy that the Ce abundance decreases by another $\sim 0.2 \mathrm{dex}$ when calculated by using the $\log (g f)$ values of the DREAM database. Hence, replacing the VALD data by the DREAM data for Ce would only increase the observed discrepancy.

(4) The predictions for the elements beyond the Ba-peak for IRAS 06530-0213 and IRAS 08143-4406 are very consistent with the observed abundances. This is an additional argument strengthening the line identification for these elements. Moreover, their abundances are very sensitive to the adopted ${ }^{13} \mathrm{C}$ pocket. Hence, these elements (especially $\mathrm{Hf}$ and $\mathrm{W}$ ) are ideally suited to discriminate between possible ${ }^{13} \mathrm{C}$ pockets. Unfortunately, a $\mathrm{Hf}$ abundance is very difficult to derive in these objects since it has only suitable lines in the blue, and the $\mathrm{W}$ abundance derived from the $5104.432 \AA$ line depends on the adopted Sm abundance (see Reyniers \& Van Winckel 2003).

\section{Conclusion}

IRAS 06530-0213 and IRAS 08143-4406 were classified as post-AGB candidates on the base of their IR properties and their spectral type. The analysis presented in this paper proved that both objects also show clear chemical evidence of their evolved, post third dredge-up character, since they show not only a clear carbon enhancement, but also a large enrichment in s-process elements. Especially IRAS 06530-0213 is an object that is very interesting for further research since it is enhanced at almost the same level as IRAS 05341+0852, the most s-process enriched object known so far. Moreover, detailed abundances of elements beyond the Ba peak were obtained, a result which was possible due to the combination of the high quality VLT+UVES spectra and newly released line data in both the VALD and the DREAM databases. The end point of the s-process $(\mathrm{Pb})$ is expected to be produced at important levels, also in these moderate metal-deficient objects, but $\mathrm{Pb}$ has no suitable lines in the optical spectrum for these temperatures and gravities.

The atmospheric and chemical properties of the two objects are remarkably similar than those of the $21 \mu \mathrm{m}$ objects. Especially the relation between third dredge-up efficiency and neutron nucleosynthesis efficiency outlined in Paper I for the $21 \mu \mathrm{m}$ stars is confirmed by these two new objects. On the other hand, the results on the two objects made the relation between metallicity and neutron nucleosynthesis efficiency even more confusing: whereas the $21 \mu \mathrm{m}$ stars of Paper I still displayed a weak correlation, the new data points (especially IRAS 06530-0213) suggest that there is no correlation at all. This means that there is a large intrinsic spread in neutron nucleosynthesis efficiency. Such a spread points to different ${ }^{13} \mathrm{C}$ pocket strengths in probably highly similar objects which is confirmed by our modelling, in which quite different ${ }^{13} \mathrm{C}$ pockets are needed to model the s-process distribution. 
A physical explanation for the different ${ }^{13} \mathrm{C}$ pocket strengths is not yet found. The proton engulfment induces an interplay between the ${ }^{14} \mathrm{~N}$ pocket, acting as a neutron poison, and the ${ }^{13} \mathrm{C}$ pocket, acting as a neutron donor. In this paper we restrengthen the observational finding that even for objects with similar metallicities, quite a different neutron irradiation is observed. Possibly rotation can play an important role in this discussion (Langer et al. 1999; Herwig et al. 2003).

Moreover, apart from the s-process enriched objects discussed in this paper, there are very similar post-AGB objects without showing any s-process enrichment. This dichotomy between s-process enriched post-AGB stars, and post-AGB stars showing no s-enrichment at all, is also not understood. Note, however, that in the FRANEC models the third dredge-up does not occur below a certain critical mass, which slightly decreases with decreasing metallicity.

It is clear that a spectroscopic infrared study of the two objects discussed in this paper would be an invaluable supplement to this optical study. Indeed, till now all enriched objects display the $21 \mu \mathrm{m}$ feature in their IR spectrum if this data is available. It should be tested if this assertion still holds after observing IRAS 06530-0213 and IRAS 08143-4406 in the IR spectral domain.

Acknowledgements. M.R. and H.V.W. acknowledge financial support from the Fund for Scientific Research - Flanders (Belgium). This research has made use of the Vienna Atomic Line Database (VALD), operated at Vienna, Austria, and the Database on Rare Earths At Mons University, operated at Mons, Belgium.

\section{References}

Anders, E., \& Grevesse, N. 1989, Geochim. Cosmochim. Acta, 53, 197

Arlandini, C., Käppeler, F., Wisshak, K., et al. 1999, ApJ, 525, 886

Balick, B., \& Frank, A. 2002, ARA\&A, 40, 439

Biémont, E., Hibbert, A., Godefroid, M., \& Vaeck, N. 1993, ApJ, 412, 431

Biémont, E., Hibbert, A., Godefroid, M., Vaeck, N., \& Fawcett, B. C. 1991, ApJ, 375, 818

Blöcker, T. 1999, in IAU Symp. 191, Asymptotic Giant Branch Stars, 21

Brix, P., \& Kopfermann, H. 1952, in Landolt-Börnstein (ed.), Zahlenwerte und Funktionen (Berlin: Springer-Verlag), I (Part 5), 1

Busso, M., Gallino, R., Lambert, D. L., Travaglio, C., \& Smith, V. V. 2001, ApJ, 557, 802

Busso, M., Gallino, R., \& Wasserburg, G. J. 1999, ARA\&A, 37, 239

Busso, M., Lambert, D. L., Beglio, L., et al. 1995, ApJ, 446, 775

Den Hartog, E. A., Curry, J. J., Wickliffe, M. E., \& Lawler, J. E. 1998, Sol. Phys., 178, 239

Gallino, R., Arlandini, C., Busso, M., et al. 1998, ApJ, 497, 388

Goriely, S., \& Mowlavi, N. 2000, A\&A, 362, 599

Gray, D. F. 1992, The observation and analysis of stellar photospheres, 2nd ed. (Cambridge University Press)
Grevesse, N., \& Sauval, A. J. 1998, Space Sci. Rev., 85, 161

Herwig, F., Langer, N., \& Lugaro, M. 2003, ApJ, 593, 1056

Hibbert, A., Biémont, E., Godefroid, M., \& Vaeck, N. 1991, A\&AS, 88,505

Holweger, H. 2001, in AIP Conf. Proc. 598: Joint SOHO/ACE workshop, Solar and Galactic Composition, 23

Hony, S., Waters, L. B. F. M., \& Tielens, A. G. G. M. 2002, A\&A, 390,533

Hrivnak, B. J., Kwok, S., \& Su, K. Y. L. 2001, AJ, 121, 2775

Hrivnak, B. J., Langill, P. P., Su, K. Y. L., \& Kwok, S. 1999, ApJ, 513, 421

Hrivnak, B. J., \& Reddy, B. E. 2003, ApJ, 590, 1049

Hu, J. Y., Te Lintel Hekkert, P., Slijkhuis, F., et al. 1994, A\&AS, 103, 301

Kupka, F., Piskunov, N., Ryabchikova, T. A., Stempels, H. C., \& Weiss, W. W. 1999, A\&AS, 138, 119

Kurucz, R. 1993, CD-ROM set, Cambridge, MA: Smithsonian Astrophysical Observatory

Kwok, S., Volk, K. M., \& Hrivnak, B. J. 1989, ApJ, 345, L51

Langer, N., Heger, A., Wellstein, S., \& Herwig, F. 1999, A\&A, 346, L37

Lawler, J. E., Bonvallet, G., \& Sneden, C. 2001a, ApJ, 556, 452

Lawler, J. E., Wickliffe, M. E., Den Hartog, E. A., \& Sneden, C. 2001b, ApJ, 563, 1075

Luck, R. E., Bond, H. E., \& Lambert, D. L. 1990, ApJ, 357, 188

Lugaro, M., Herwig, F., Lattanzio, J. C., Gallino, R., \& Straniero, O. 2003, ApJ, 586, 1305

Malaney, R. A. 1987, Ap\&SS, 137, 251

Meixner, M., Ueta, T., Dayal, A., et al. 1999, ApJS, 122, 221

Preite-Martinez, A. 1988, A\&AS, 76, 317

Reddy, B. E., \& Parthasarathy, M. 1996, AJ, 112, 2053

Reimers, D. 1975, Circumstellar envelopes and mass loss of red giant stars, Problems in stellar atmospheres and envelopes (New York: Springer-Verlag), 229

Reyniers, M. 2002, Ph.D. Thesis, K.U. Leuven

Reyniers, M., \& Van Winckel, H. 2001, A\&A, 365, 465

Reyniers, M., \& Van Winckel, H. 2003, A\&A, 408, L33

Reyniers, M., Van Winckel, H., Biémont, E., \& Quinet, P. 2002, A\&A, 395, L35

Sahai, R. 2001, in Post-AGB Objects as a Phase of Stellar Evolution, 53

Slijkhuis, S. 1992, Ph.D. Thesis, University of Amsterdam

Sneden, C., Lawler, J. E., \& Cowan, J. J. 2002, Phys. Scr., 100, 15

Sneden, C. A. 1973, Ph.D. Thesis, University of Texas at Austin

Straniero, O., Chieffi, A., Limongi, M., et al. 1997, ApJ, 478, 332

Straniero, O., Dominguez, I., Cristallo, S., \& Gallino, R. 2003, PASA, 20 , in press

Szczerba, R., Górny, S. K., \& Zalfresso-Jundziłło, M. 2001, in PostAGB Objects as a Phase of Stellar Evolution, 13

Ueta, T., Meixner, M., \& Bobrowsky, M. 2000, ApJ, 528, 861

Van Winckel, H. 1997, A\&A, 319, 561

Van Winckel, H. 2003, ARA\&A, 41, 391

Van Winckel, H., \& Reyniers, M. 2000, A\&A, 354, 135 (Paper I)

Van Winckel, H., Waelkens, C., \& Waters, L. B. F. M. 1995, A\&A, 293, L25

von Helden, G., Tielens, A. G. G. M., van Heijnsbergen, D., et al. 2000, Science, 288, 313 